\title{
Sparse Antenna Array Design for MIMO Radar Using Multiobjective Differential Evolution
}

\author{
Zhi-Kun Chen, Feng-Gang Yan, Xiao-Lin Qiao, and Yi-Nan Zhao \\ School of Electronics and Information Engineering, Harbin Institute of Technology, Harbin Heilongjiang 150001, China \\ Correspondence should be addressed to Xiao-Lin Qiao; paulqiaohit@163.com
}

Received 30 June 2016; Revised 20 October 2016; Accepted 7 November 2016

Academic Editor: Sotirios K. Goudos

Copyright (C) 2016 Zhi-Kun Chen et al. This is an open access article distributed under the Creative Commons Attribution License, which permits unrestricted use, distribution, and reproduction in any medium, provided the original work is properly cited.

\begin{abstract}
A two-stage design approach is proposed to address the sparse antenna array design for multiple-input multiple-output radar. In the first stage, the cyclic algorithm (CA) is used to establish a covariance matrix that satisfies the beam pattern approximation for a full array. In the second stage, a sparse antenna array with a beam pattern is designed to approximate the desired beam pattern. This paper focuses on the second stage. The optimization problem for the sparse antenna array design aimed at beam pattern synthesis is formulated, where the peak side lobe (PSL) is weakly constrained by the mean squared error. To solve this optimization problem, the differential evolution (DE) algorithm with multistrategy is introduced and PSL suppression is treated as an inequality constraint. However, in doing so, a new multiobjective optimization problem is created. To address this new problem, a multiobjective differential evolution algorithm based on Pareto technique is proposed. Numerical examples are provided to demonstrate the advantages of the proposed approach over state-of-the-art methods, including DE and genetic algorithm.
\end{abstract}

\section{Introduction}

By exploiting the diverse waveform from its multiple antennas, multiple-input multiple-output (MIMO) radar transmits a probing waveform that can be chosen at will to maximize the power around areas of interest or approximate a desired beam pattern [1]. The transmitting beam pattern design for MIMO radar has become a popular research topic in recent years $[2,3]$. This topic has been extensively investigated, and numerical algorithms that involve synthesizing the covariance matrix and waveform design have been proposed. One popular and very effective approach for processing a desired beam pattern is based on the convex optimization (CVX) principle and solved by the semidefinite quadratic programming (SQP) question [3]. Another approach is the cyclic algorithm (CA), which optimally matches the designed and desired beam patterns via iteration [4].

Sparse antenna array has several advantages in the highresolution thinned configurations for phased array radar [57]. MIMO radar also uses such array to obtain additional degrees of freedom $[4,8-10]$. However, because of array thinning, the side lobe level increases and subsequently leads to the grating lobe. In this case, maintaining the approximation performance and preventing the grating lobe present two major challenges for a MIMO radar with sparse antenna arrays. Roberts applied CA in the sparse antenna array design for a MIMO radar to approximate a desired beam pattern [4]. However, unlike other beam pattern synthesis methods, this algorithm only synthesizes the beam pattern for a single target location $[2,3]$. For wide beam pattern synthesis, an integrated optimization of beam pattern synthesis and sparse antenna array design called CVXGA is proposed in our previous work [10]. This method uses SQP for wide beam pattern synthesis and applies the genetic algorithm (GA) for the sparse array design. However, given the convergence rate of GA and the high peak side lobe (PSL) of its results, CVXGA is not desirable for the sparse antenna array design of MIMO radar. Compared with GA, the differential evolution (DE) algorithm fastens the convergence rate and solves the optimization problems using several techniques, including mutation, selection, and crossover. DE has been utilized in the antenna array synthesis of phased array radar but is yet to be applied for MIMO radar [11-13]. To prevent high PSL, the sparse antenna array design for MIMO radar must 
be formulated as a constrained optimization problem where PSL suppression is considered an inequality constraint. Both GA and DE are single-objective evolutionary algorithms that cannot synthesize beam pattern and suppress PSL simultaneously. To solve this constrained optimization problem, a penalty method is applied by using a fitness function that is modified based on the penalty function [14]. However, most penalty methods require highly complex dependent parameters that result in a performance decline.

According to [15], the optimization problem with inequality constraint can be transformed into a multiobjective optimization problem that can be solved using the multiobjective differential evolution (MODE) algorithm. This work attempts to optimize the sparse antenna array design of MIMO radar based on this idea. Specifically, DE is applied to the sparse array design aimed at beam pattern synthesis, while MODE based on Pareto optimal solutions is applied to the sparse array design aimed at both beam pattern synthesis and PSL suppression. The performances of GA in [10], DE, and MODE are then compared via numerical simulations.

The rest of this paper is organized as follows. In Section 2, the signal model for beam pattern synthesis and the sparse antenna array design problem are introduced. In Section 3, two evolution algorithms, namely, DE and MODE, are presented to solve the optimization problem of the sparse antenna array design. Numerical simulations are presented in Section 4, while the conclusions are presented in Section 5.

\section{Signal Model and Problem Statement}

Consider a uniform linear array (ULA) composed of $N$ antennas with a length of $(N-1) \times d$, where $d$ refers to the separation between transmitting antennas. The steering vector is expressed as follows:

$$
\mathbf{a}(\theta)=\left[1, e^{j 2 \pi d \sin (\theta) / \lambda}, \ldots, e^{j 2 \pi d(N-1) \sin (\theta) / \lambda}\right]^{T},
$$

where $\lambda$ denotes the wavelength of the narrowband waveform and $d=\lambda / 2$. Let the angular scanning grid be spanned by $\left\{\theta_{k}\right\}_{k=1}^{K}$; then the set of steering vectors can be collected into the steering matrix $\mathbf{A} \in \mathbb{C}^{N \times K}$

$$
\mathbf{A}=\left[\begin{array}{llll}
\mathbf{a}\left(\theta_{1}\right) & \mathbf{a}\left(\theta_{2}\right) & \cdots & \mathbf{a}\left(\theta_{K}\right)
\end{array}\right]
$$

2.1. Beam Pattern Synthesis. Let $\mathbf{v}(l) \in \mathbb{C}^{N \times 1}$ denote the $l$ th transmitted signal pulse. The covariance matrix of the transmitted waveforms is computed as follows:

$$
\mathbf{R}=E\left\{\mathbf{v}(l) \mathbf{v}^{H}(l)\right\},
$$

where $\mathbf{R} \in \mathbb{C}^{N \times N}$ and $E\{\cdot\}$ denotes the expectation operator. The transmitting beam pattern at $\theta_{k}$ can be defined as follows:

$$
p\left(\theta_{k}\right)=\mathbf{a}^{H}\left(\theta_{k}\right) \mathbf{R a}\left(\theta_{k}\right) .
$$

Therefore, the transmitting beam pattern for each angle $\left\{\theta_{k}\right\}_{k-1}^{K}$ can be collected in its vector form as follows:

$$
\mathbf{p}=\left[p\left(\theta_{1}\right) \cdots p\left(\theta_{k}\right)\right]^{T}=\operatorname{diag}\left[\mathbf{A}^{H} \mathbf{R A}\right],
$$

where $\operatorname{diag}(\cdot)$ is a diagonal matrix that is formed from the elements of some square matrix. Similar to (5), a desired transmitting beam pattern is defined as follows:

$$
\mathbf{p}_{d}=\left[\phi\left(\theta_{1}\right), \ldots, \phi\left(\theta_{K}\right)\right]^{T},
$$

where $\phi(\theta)$ is the desired beam pattern at angle $\theta$. The optimization problem of beam pattern approximation can be formulated as follows:

$$
\begin{array}{ll}
\min _{\mathbf{R}} & \left\|\operatorname{diag}\left(\mathbf{A}^{H} \mathbf{R A}\right)-\mathbf{p}_{d}\right\|^{2}, \\
\text { s.t. } & \mathbf{a}^{H}\left(\theta_{c}\right) \mathbf{R a}\left(\theta_{c}\right)=1 \\
& \mathbf{R} \geq 0,
\end{array}
$$

where $\|\cdot\|$ refers to the Frobenius norm operation for the matrix. As shown in (7), beam pattern synthesis aims to design a matching beam pattern to approximate the desired transmitting beam pattern in the whole area. The mean squared error (MSE) between the designed and desired beam pattern must be maintained as low as possible to evaluate the approximation performance. The MSE can be computed as follows:

$$
\mathrm{MSE}=\left\|\mathbf{p}-\mathbf{p}_{d}\right\|^{2} .
$$

According to $[4,16]$, the optimum $\mathbf{R}$ for beam pattern synthesis can be obtained using SQP and CA.

2.2. Sparse Antenna Array Design for MIMO Radar. For the sparse antenna array design, only the $M<N$ antennas are assumed to be available in $N$ candidate positions of the transmitter. The pattern $\mathbf{p}$ can be defined as follows:

$$
\mathbf{p}=\mathbf{a}_{S}^{H}\left(\theta_{k}\right) \mathbf{R} \mathbf{a}_{S}\left(\theta_{k}\right)
$$

where $\mathbf{a}_{S} \in \mathbb{C}^{N \times 1}$ denotes the steering vector of the sparse antenna array. The main purpose of the sparse antenna array design is to determine a set of $M$ antenna positions in the $N$ candidate position. Following [4], the steering vector of sparse array $\mathbf{a}_{S}$ can be treated as a vector that contains only the element of a that corresponds to the chosen locations of $N$ antennas. The vector $\mathbf{p}_{S}$ is introduced to represent the antenna position as a set of binary codes containing the values of 0 and 1, where 1 indicates that the antenna element with an index corresponding to the location of 1 in $\mathbf{p}_{S}$ belongs to the sparse antenna array while 0 indicates otherwise. The vector $\mathbf{a}_{S}$ is obtained as follows based on the relationship between a and $\mathbf{p}_{S}$ :

$$
\mathbf{a}_{S}=\mathbf{p}_{S} \odot \mathbf{a},
$$

where $\odot$ denotes the Hadamard elementwise product. The steering matrix of the sparse antenna array can be expressed as follows:

$$
\mathbf{A}_{S}=\left[\begin{array}{llll}
\mathbf{a}_{S}\left(\theta_{1}\right) & \mathbf{a}_{S}\left(\theta_{2}\right) & \cdots & \mathbf{a}_{S}\left(\theta_{K}\right)
\end{array}\right] .
$$
for MIMO Radar. For the beam pattern synthesis [16], 


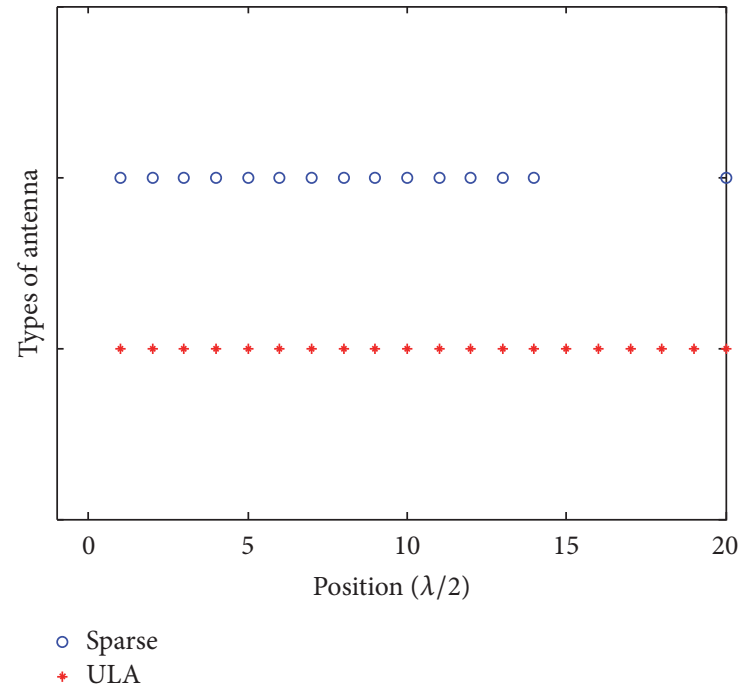

(a) Position of antenna

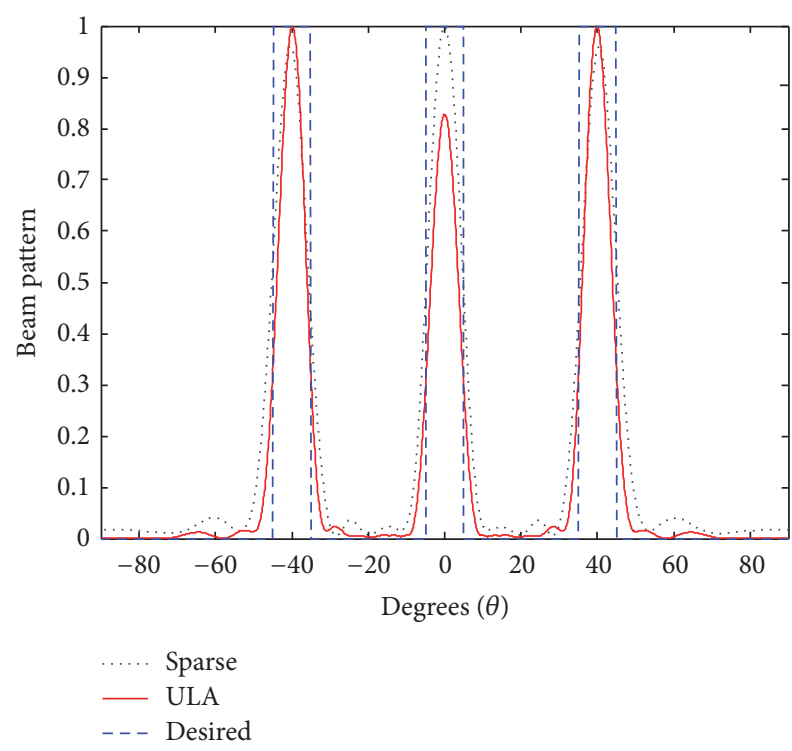

(b) Transmit beam pattern

FIGURE 1: Results of the sparse antenna array using CA+CA, $M=15$, and $N=20$. The desired beam pattern is represented by the blue dashed line.

directional wide beam pattern and multimain lobes with a highly complex covariance matrix $\mathbf{R}$ are adopted for a MIMO radar. As shown in [4], the CA is applied repeatedly to solve the optimization problem in the two-stage design. To prevent confusion, this approach is represented as CA+CA in the rest of this paper. In the first stage, a weighted matrix $\mathbf{W}$ that satisfies the beam pattern approximation for a full array is synthesized. Equation (7) can be decomposed as follows:

$$
\begin{array}{ll}
\min _{\mathbf{W}} & \left\|\mathbf{W}^{H} \mathbf{A}-\mathbf{U}\right\| \\
\text { s.t. } & \operatorname{diag}\left(\mathbf{U}^{H} \mathbf{U}\right)=\mathbf{p}_{d}, \\
& \mathbf{W} \geq 0,
\end{array}
$$

where $\mathbf{W}=\mathbf{R}^{1 / 2}$. In the second stage, using the $\mathbf{W}$ and candidate positions provided by a full array, the element positions of the sparse antenna array are optimized to maintain the beam pattern approximation performance as much as possible. The sparse antenna array design can be expressed with given $\mathbf{W}$ as follows:

$$
\begin{array}{ll}
\min _{\mathbf{p}_{S}} & \left\|\mathbf{W}^{H} \mathbf{A}_{S}-\mathbf{U}\right\| \\
\text { s.t. } & \operatorname{diag}\left(\mathbf{U}^{H} \mathbf{U}\right)=\mathbf{p}_{\mathrm{d}} \\
\mathbf{a}_{S}= & \mathbf{p}_{S} \odot \mathbf{a} .
\end{array}
$$

Given its initial position, $\mathbf{p}_{S}$ is optimized to match a desired transmitting beam pattern via random perturbation. $\mathrm{CA}$ is applied for the sparse antenna array design aimed at beam pattern synthesis, and the results are presented in Figure 1.

The simulation results show that $\mathrm{CA}+\mathrm{CA}$ can generate a sparse antenna array with a matching beam pattern. This array can be regarded as a $N-1$ dimensional ULA with one antenna element located at the maximum aperture as shown in Figure 1(a). Therefore, despite its excellent approximation performance, $\mathrm{CA}+\mathrm{CA}$ is unsuitable for the sparse antenna array design for MIMO radar.

\section{Sparse Antenna Array Design for MIMO Radar}

A new formula for the sparse antenna array design for MIMO radar aimed at beam pattern synthesis is proposed. Given its waveform diversity, the optimization problem for beam pattern synthesis cannot be solved in the process of sparse antenna array design, which is unlike the phased array radar. Following $[4,10]$, a two-stage design approach is adopted for the sparse antenna array design for MIMO radar. In the first stage, the covariance matrix $\mathbf{R}$ is synthesized to generate a matching beam pattern for a full array with an $N$ antenna element. As mentioned above, CA and SQP are two highly effective algorithms, but a different algorithm for sparse antenna array design must be selected. In the second stage, the element positions of the full array are treated as candidate positions that are chosen by a sparse antenna array with $M$ antenna elements. Accordingly, this work only focuses on the latter stage.

To control the designed beam pattern, the optimization problem for a sparse antenna array design aimed at beam pattern synthesis can be written as follows:

$$
\begin{array}{ll}
\min _{x} & f(x) \\
\text { s.t. } & \mathbf{p}_{S}(1)=1, \\
& \mathbf{p}_{S}(N)=1,
\end{array}
$$


where $\mathbf{p}_{S}(1)=1$ and $\mathbf{p}_{S}(N)=1$ constrain the same antenna aperture as the full array in the first stage, while the objective function $f(x)$ is expressed as follows:

$$
f(x)=\frac{1}{K} \sum_{k=1}^{K}\left[\phi\left(\theta_{k}\right)-\operatorname{diag}\left(\mathbf{a}_{S}^{H}\left(\theta_{k}\right) \mathbf{R} \mathbf{a}_{S}\left(\theta_{k}\right)\right)\right]^{2},
$$

where $\mathbf{R}$ is obtained from the first stage and $f(x)$ represents the MSE between the beam pattern of a sparse antenna array and the desired beam pattern. The above function weakly constrains the PSL in the whole area. To avoid the local optimum as shown in Figure 1 and to achieve faster convergence with respect to GA as shown in [10], DE with multistrategy is introduced to solve this singleobjective optimization problem and $f(x)$ is treated as the fitness function. The goal of the optimization is to minimize the fitness value or $f(x)$ by finding the optimum element positions.

3.1. Sparse Antenna Array Using DE with Multistrategy. DE is a powerful stochastic algorithm that evolves a population of $N P$ - and $N$-dimensional individuals toward the global optimum $[13,17]$. Previous studies have mostly synthesized a sparse antenna array for a phased array radar [10, 13, 14]. This work promotes the application of DE in MIMO radar and exploits its constraint optimization ability.

Initialization. Given that the antenna element is placed at both ends of the array, only $D=N-2$ variables in the individual need to be evolved. Assuming that Max_Iteration is the maximum iteration number of the evolution process, the initial value of the $j$ parameter in the $i$ th population at generation $G$ can be written as follows:

$$
\begin{aligned}
& x_{i, G}^{j}=\operatorname{rand}(0,1) \cdot\left(x_{\max }^{j}-x_{\min }^{j}\right)+x_{\min }^{j}, \\
& \quad i=1, \ldots, N P, j=1, \ldots, D,
\end{aligned}
$$

where $D=N-2, G \in[1, \ldots$, Max_Iteration $]$, and $\operatorname{rand}(0,1)$ denotes a uniformly distributed random variable within the range $[0,1]$ and $x_{\max }^{j}$ and $x_{\text {min }}^{j}$ are the lower and upper bounds of the $j$ th variable parameter, respectively. The individual in the $i$ th population at generation $G$ can be expressed in its vector form as follows:

$$
\begin{aligned}
& \mathbf{x}_{i, G}=\left[x_{i, G}^{1}, x_{i, G}^{2}, \ldots, x_{i, G}^{D}\right] \\
& i=1, \ldots, N P, j=1, \ldots, D .
\end{aligned}
$$

Coding. To locate the array element in $\mathbf{p}_{S}$, the real variable must be converted into a binary coded variable. Therefore, the initial value of the antenna position is set as the partition points of a specified antenna aperture. The random perturbation is dominated by $\mathbf{x}_{i, G}$, which is binary coded in $\mathbf{p}_{S}$ as follows:

$$
\mathbf{p}_{S}(n)=\text { binary } \operatorname{sort}(\mathbf{x}(n))^{T}, \quad n=2, \ldots, N-1,
$$

where binary $\operatorname{sort}(\cdot)$ denotes the real variables that are sorted by size as integer variables converted into binary codes. In this case, $\mathbf{p}_{S}(1)$ is equal to $1, \mathbf{p}_{S}(N)$ is equal to 1 , and $\mathbf{p}_{S}(2: N-1)$ is presented by (18). The sum of 1 in the vector $\mathbf{p}_{S}$ is equal to $M$ for the predefined number antenna elements of a sparse antenna array.

Mutation. The mutation operator produces a mutant vector $\mathbf{u}_{i, G}=\left(u_{i, G}^{1}, u_{i, G}^{2}, \ldots, u_{i, G}^{D}\right)$ with respect to each individual $\mathbf{x}_{i, G}$ at the generation $G$. The following mutation strategies are often applied in traditional DE [18]:

(1) DE/rand/1.bin: $\mathbf{u}_{i, G}=\mathbf{x}_{r 1, G}+F \cdot\left(\mathbf{x}_{r 2, G}-\mathbf{x}_{r 3, G}\right)$,

(2) DE/best/1.bin: $\mathbf{u}_{i, G}=\mathbf{x}_{\text {Best }, G}+F \cdot\left(\mathbf{x}_{r 1, G}-\mathbf{x}_{r 2, G}\right)$,

(3) DE/rand-to-best/1: $\mathbf{u}_{i, G}=\mathbf{x}_{i, G}+F \cdot\left(\mathbf{x}_{\text {Best }, G}-\mathbf{x}_{i, G}\right)+F \cdot$ $\left(\mathbf{x}_{r 1, G}-\mathbf{x}_{r 2, G}\right)$,

(4) DE/best $/ 2: \mathbf{u}_{i, G}=\mathbf{x}_{\mathrm{Best}, G}+F \cdot\left(\mathbf{x}_{r 1, G}-\mathbf{x}_{r 2, G}\right)+F \cdot\left(\mathbf{x}_{3, G}-\right.$ $\left.\mathbf{x}_{4, G}\right)$,

(5) $\mathrm{DE} / \mathrm{rand} / 2: \mathbf{u}_{i, G}=\mathbf{x}_{r 1, G}+F \cdot\left(\mathbf{x}_{r 2, G}-\mathbf{x}_{r 3, G}\right)+F \cdot\left(\mathbf{x}_{4, G}-\right.$ $\left.\mathbf{x}_{5, G}\right)$,

where $r 1, r 2, r 3, r 4, r 5 \in[1, N P], r 1 \neq r 2 \neq r 3 \neq r 4 \neq r 5 \neq i$, and $\mathbf{x}_{\mathrm{Best}, G}$ is the individual with the minimum fitness value in the population at generation $G$. The scaling factor $F \in[0,2]$ is constant.

Crossover. A trial vector is produced by vector $\mathbf{x}_{i, G}$ and its corresponding mutant vector $\mathbf{v}_{i, G}$. The basic crossover strategy is defined as follows:

$$
v_{i, G}^{j}= \begin{cases}u_{i, G}^{j} & \text { if } \operatorname{rand}(0,1) \leq \mathrm{CR} \text { or } j=j_{\text {rand }} \\ x_{i, G}^{j} & \text { otherwise. }\end{cases}
$$

The crossover factor CR is a user-specified constant that satisfies $C R \in[0,1]$ and controls the fraction of parameter values copied from the mutant vector.

Selection. The objective function value of each trial vector $f\left(\mathbf{v}_{i, G}\right)$ is compared with that of its corresponding target vector $f\left(\mathbf{x}_{i, G}\right)$. The vector with the smaller fitness value will be retained in the next generation. The operation can be expressed as follows:

$$
\mathbf{v}_{i, G+1}= \begin{cases}\mathbf{v}_{i, G} & \text { if } f\left(\mathbf{v}_{i, G}\right)<f\left(\mathbf{x}_{i, G}\right) \\ \mathbf{x}_{i, G} & \text { otherwise. }\end{cases}
$$

Afterward, we calculate the fitness function and repeat the selection, crossover, and mutation processes per generation until a specific stopping criterion is satisfied. Algorithm 1 presents the DE algorithm implementation steps in detail.

3.2. Sparse Antenna Array Design Using MODE. To obtain the desired PSL and improve the robustness of DE for the sparse antenna array design, PSL suppression is regarded as an inequality constraint in the optimization problem as defined in (14) [18]. PSL level (PSLL) indicates the amplitude of the highest PSL outside of the desired beam pattern [11, 19]. The optimization problem for the sparse antenna array design 


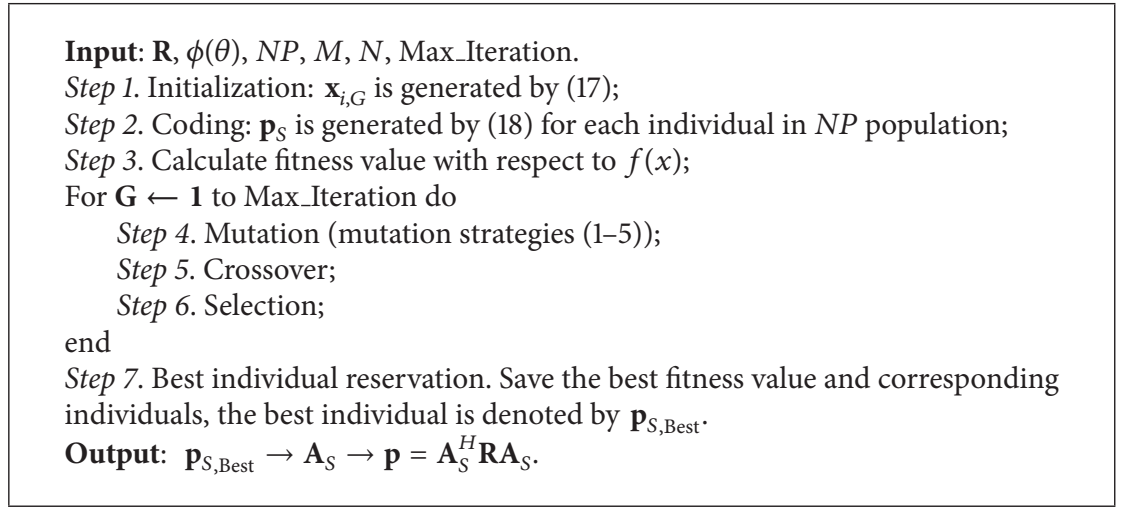

Algorithm 1: Sparse antenna array design using DE algorithm with multistrategy.

aimed at both beam pattern synthesis and PSL suppression can be formulated as follows:

$$
\begin{array}{ll}
\min _{x} & f(x) \\
\text { s.t. } & \mathbf{p}_{S}(1)=1, \\
& \mathbf{p}_{S}(N)=1 \\
& \text { PSLL }-\delta<0,
\end{array}
$$

where $\delta$ is the tolerance value for PSLLs. Following the idea of multiobjective optimization, the inequality constraint can be regarded as another objective function in the evolution process [20]. This constraint can be optimized in the parallel implementation as follows:

$$
\begin{array}{ll}
\min _{x} & \left(f_{1}(x), f_{2}(x)\right) \\
\text { s.t. } & \mathbf{p}_{S}(1)=1, \\
& \mathbf{p}_{S}(N)=1,
\end{array}
$$

where $f_{1}(\mathbf{x})$ is defined in (15) and $f_{2}(\mathbf{x})=$ PSLL. This optimization problem can be easily solved using MODE, which is designed to handle a multiple set of solutions in a single iteration.

In the multiobjective domain, the evolutionary algorithm aims to identify a set of Pareto optimal solutions to operate the selection of the best individual for mutation [20]. At the end of the evolutionary search, the nondominated solution archive passes through a Pareto dominance to yield the global near-Pareto optimal frontier.

Definition 1 (opposition-based learning). Opposition-based learning is used to generate opposite solutions in the initialization to increase the chances of starting with the fittest solution [21], which can be expressed as follows:

$$
\begin{aligned}
y_{i}^{j}=\left(x_{\max }^{j}+x_{\min }^{j}\right)-x_{i}^{j}, & \\
& i=1, \ldots, N P, j=1, \ldots, D .
\end{aligned}
$$

Definition 2 (Pareto dominance). A solution $x_{1}$ is said to dominate another solution $x_{2}$ under the following conditions:

(1) $\forall m \in\left(1, \ldots, M_{\mathrm{obj}}\right): f_{m}\left(x_{1}\right) \leq f_{m}\left(x_{2}\right)$,

(2) $\exists m \in\left(1, \ldots, M_{\mathrm{obj}}\right): f_{m}\left(x_{1}\right)<f_{m}\left(x_{2}\right)$,

where $M_{\mathrm{obj}}$ is the number of objective functions and $f_{m}(\cdot)$ is the corresponding fitness function. Any individual that is not dominated by any other member is considered nondominated.

Definition 3 (fast nondominated sorting). Assume a Pareto optimal set denoted by $S$. $n_{p}$ denotes the number of dominated solutions, while $S_{p}$ is a set of solutions dominated by the solution $p$ [21].

For every solution $p$ in $S$, both $n_{p}$ and $S_{p}$ are calculated. All solutions in the first nondominated front $F_{1}$ clear their domination count to zero. Afterward, when $n_{p}=0$, each solution $p$ visits each member $q$ of its set $S_{p}$ and $n_{p}=$ $n_{p}-1$. Any member $q$ is saved in a separate list $P$. These members belong to the second nondominated front $F_{2}$. Each member of $P$ and the third front $F_{3}$ are identified. This process continues until all fronts have been identified.

In our approach, $M_{\mathrm{obj}}=2$, and fast nondominated sorting is applied to guarantee that the population maintains its original size and that the nondominated solutions in the population are identified at each generation of the evolutionary process. The nondominated solutions are saved in the advanced population that corresponds to the feasible solution [22]. Otherwise, the infeasible solution is reserved in the current population. Some steps in MODE are applied similarly as those in DE, including coding and crossover. Therefore, the steps of MODE for the sparse antenna array design are presented in Algorithm 2.

\section{Numerical Example}

Several numerical examples are provided to validate the effectiveness of the proposed method. For each example, $M=$ 15 antenna elements of $N=20$ candidate positions (with a total aperture length of $20 \times d=10 \lambda$ and sparse rate of 
Input: $\mathbf{R}, \phi(\theta), N P, M, N$, Max_Iteration.

Step 1. Initialization: Generate NP random solutions using (16), and generate NP opposite solutions using opposition-based learning.

Step 2. Coding: $\mathbf{p}_{S}$ is generated using (18) for each solutions in these $2 N P$ solutions;

Step 3. Calculate fitness value: $f_{1}(x)$ is defined by $(15), f_{2}(x)=\operatorname{PSLL}(x)$, evaluate the fitness value at these $2 N P$ solutions, select the

$N P$ fittest solution via fast non-dominated sorting,

$F E S=N P$, and store the solutions in the current population pop_c.

For FES $\leftarrow 1$ to Max_Iteration do

For $i \leftarrow 1$ to $N P$ do

Step 4. Mutation: Randomly select three distinct individuals, $\mathbf{x}_{r 1}, \mathbf{x}_{r 2}$, and $\mathbf{x}_{r 3}$,

who are all different from the target individual. $\mathbf{x}_{t b, G}$ denotes the best

individuals among the three which is mean that the one has best

fitness function value. Generate a perturbed individual $\mathbf{u}_{i}$ as follows:

Step 5. Crossover: FES $=$ FES +1 ;

$$
\mathbf{u}_{i, G+1}=\mathbf{x}_{t b, G}+F\left(\mathbf{x}_{r 2, G}+\mathbf{x}_{r 3, G}\right)
$$

Step 6. Pareto dominance

if $\left(\mathbf{v}_{i}\right.$ dominates $\left.\mathbf{x}_{i}\right)$

replace $\mathbf{x}_{i}$ by $\mathbf{v}_{i}$ in the current population pop_c, and then add $\mathbf{x}_{i}$ to the advanced population pop_a.

else

add $\mathbf{v}_{i}$ to the advanced population pop $\_$.

End End

Step 7. Select the NP fittest solutions via fast non-dominated sorting and save

End

them in the pop_c $; \mathbf{p}_{S, \text { Best }}$ denotes the best individual with respect to $f_{1}(x)$.;

Output: $\mathbf{p}_{S, \text { Best }} \rightarrow \mathbf{A}_{S} \rightarrow \mathbf{p}=\mathbf{A}_{S}^{H} \mathbf{R} \mathbf{A}_{S}$.

Algorithm 2: Sparse antenna array design using MODE.

$75 \%)$ are used. The entire angle area is $\left(-90^{\circ}, 90^{\circ}\right)$ with a $0.1^{\circ}$ angular spacing (in this case, $K=1801$ ). To highlight the approximation performance, the central angle of the desired beam pattern is expressed as $\theta_{c}=\left[\begin{array}{lll}-40^{\circ} & 0^{\circ} & 40^{\circ}\end{array}\right]$, and the beam width is expressed as $\Delta=10^{\circ}$

$$
\phi(\theta)= \begin{cases}1, & \theta \in\left[\theta_{c}-\frac{\Delta}{2}, \theta_{c}+\frac{\Delta}{2}\right] \\ 0, & \text { otherwise. }\end{cases}
$$

For clarity, the chosen antennas are shown along with the ULA $(N=20)$. The parameters are defined and applied uniformly as follows:

(1) population size: $N P=100$,

(2) initial range: $x_{\max }=1, x_{\min }=0$,

(3) mutation probability: $F=0.8$,

(4) crossover probability: $\mathrm{CR}=0.3$,

(5) maximum number of iterations: Max_Iteration = 200.

4.1. Example 1: Algorithm Selection for Beam Pattern Synthesis in the First Stage. In this example, two effective algorithms (CVX and CA) for evaluating beam pattern approximation performance are compared, and the suitable algorithm is selected to establish the covariance matrix $\mathbf{R}$ in the first stage. For this purpose, a sparse antenna array is generated in the second stage by applying GA under the simulation condition. This approach is represented as SQP+GA in the remaining parts of this paper.

As described in [10], selected $\mathbf{R}$ is modeled as an SQP and solved by a SeDuMi solver [23]. GA is then applied to the sparse antenna array design. We present the results along with the ULA $(N=15)$ to show that the additional degrees of freedom provided by a sparse antenna array can indeed benefit a MIMO radar.

Figure 2 shows the sparse antenna array and transmitting beam pattern that are obtained using GA with $\mathbf{R}$ provided by SQP. The transmitting beam pattern obtained using GA has a lower approximation performance than that obtained using CA (Figure 1) for a full array. Therefore, a poor approximation performance for the sparse antenna array is obtained, and the grating lobes are formed outside of the desired beam pattern. In other words, SQP+GA cannot provide a sparse antenna array with a sufficient approximate beam pattern for MIMO radar because its high PSLLs will result in beam pattern degradation.

$\mathrm{CA}$ is then used to provide the optimum covariance matrix $\mathbf{R}$ for GA (CA+GA). Figure 3 presents the results.

Figure 3 shows that the transmitting beam pattern obtained using $\mathrm{CA}+\mathrm{GA}$ with $M=15$ has a much better approximation performance than that obtained using SQP+GA because the CA offers advantages in highresolution beam pattern synthesis based on alternating projection. To analyze the performance of these two algorithms, 

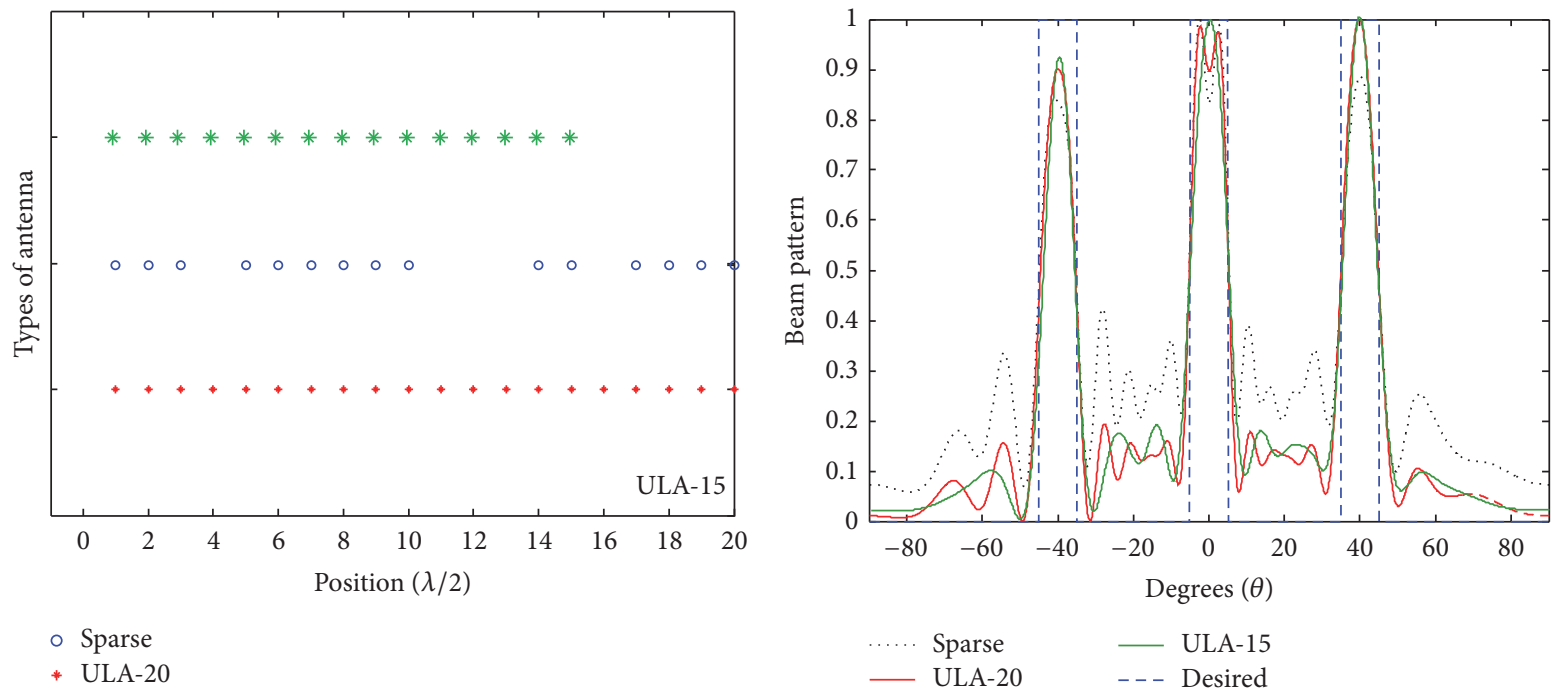

(a) Position of antenna

(b) Transmitting beam pattern

FIGURE 2: Results of SQP+GA in [8, 9].

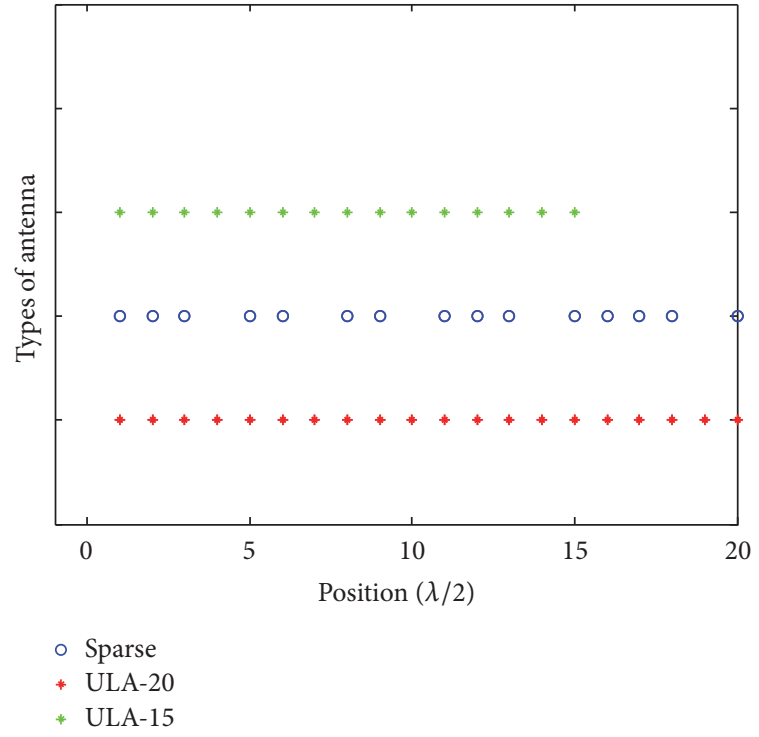

(a) Position of antenna

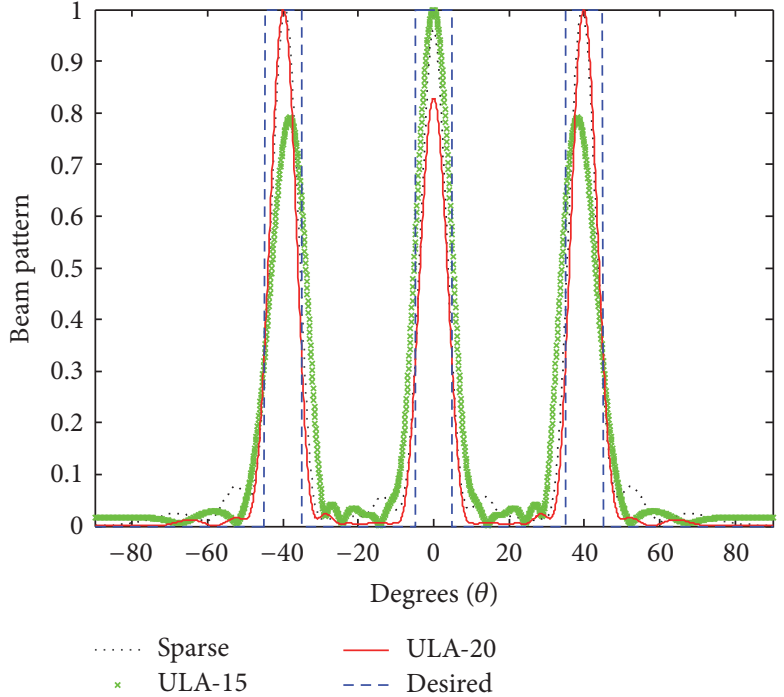

(b) Transmitting beam pattern

Figure 3: Results of CA+GA.

we compare MSE with PSLL in Table 1 and then list the iterations needed for the convergence and runtime for GA.

Table 1 shows that $\mathrm{CA}+\mathrm{GA}$ outperforms SQP+GA in both MSE and PSLL. Therefore, CA is used to establish the covariance matrix $\mathbf{R}$ in the following simulations. GA still converges to the result after more than 100 iterations.

These results also prove that the beam pattern of the sparse antenna array improves the approximation performance of ULA with the same array elements (ULA with $N=15$ ) by expanding the antenna aperture, but such performance remains inferior to that of ULA with the same antenna aperture (ULA with $N=20$ ) because of the missing antenna element.
4.2. Example 2: Sparse Antenna Array Design Using DE in the Second Stage. In this example, DE with multistrategy is applied to optimize the sparse antenna array for MIMO radar. CA is reapplied to establish the optimum covariance matrix $\mathbf{R}$, and the evolution process continues until the maximum number of iterations for testing convergence has been reached.

Figures 4-8 show the chosen antenna positions and the designed beam pattern. The sparse antenna array obtained using DE with multistrategy can maintain the beam pattern approximation performance. However, the beam pattern in the area of the main lobes and the PSLLs outside of the beam pattern are balanced. The PSLLs increase as the match 
TABLE 1: Comparison of SQP and CA.

\begin{tabular}{lcccc}
\hline Algorithm & & \multicolumn{3}{c}{ Performance } \\
& MSE & PSLLs & Iterations & Runtime (min/s) \\
\hline CA for ULA with $N=15$ & 0.1355 & 0.0405 & $/$ & $/$ \\
CA for ULA with $N=20$ & 0.0831 & 0.0232 & $/$ & $/$ \\
SQP for ULA with $N=15$ & 0.2617 & 0.1863 & $/$ & $/$ \\
SQP for ULA with $N=20$ & 0.1927 & 0.1803 & 98 & $7 / 04$ \\
SQP+GA & 0.4867 & 0.4326 & 102 & $7 / 08$ \\
CA+GA & 0.0920 & 0.0245 & $/$ \\
\hline
\end{tabular}

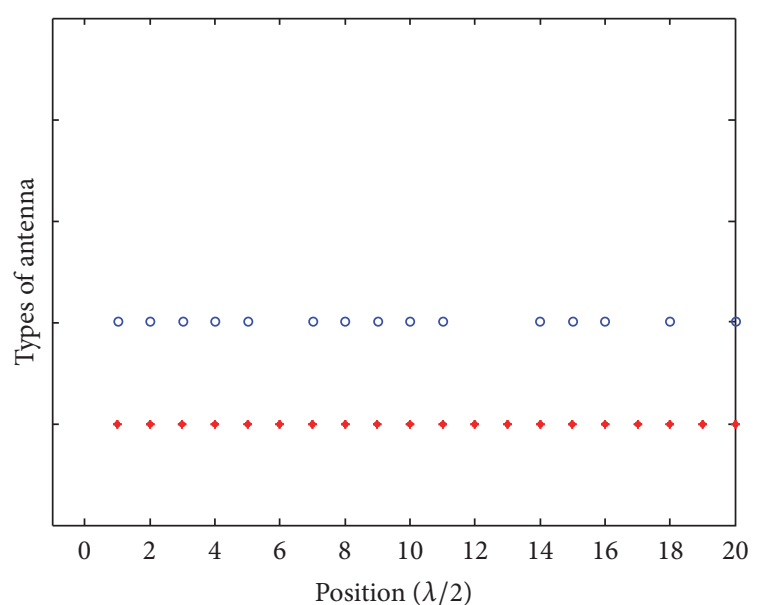

- Sparse

* ULA

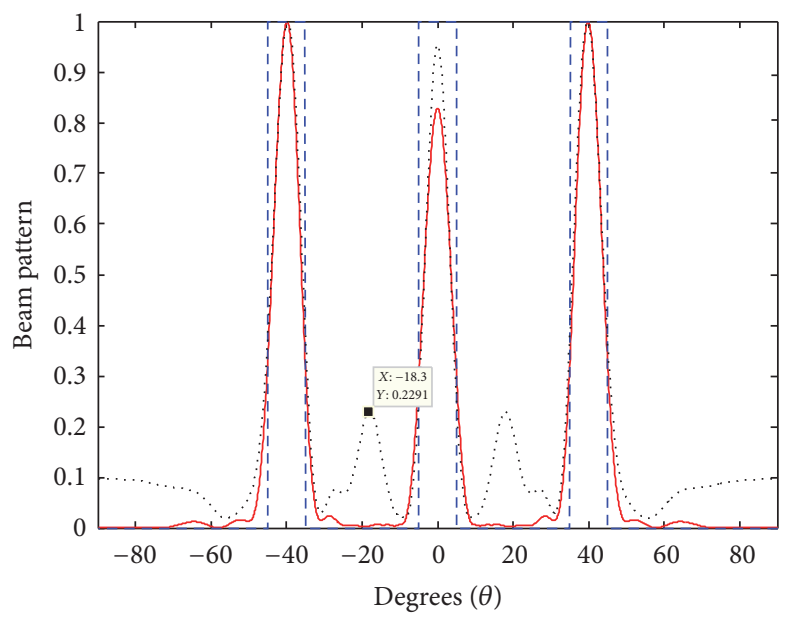

Sparse

- ULA

(b) Transmitting beam pattern

(a) Position of antenna

FIGURE 4: Results of DE with mutation strategy 1.

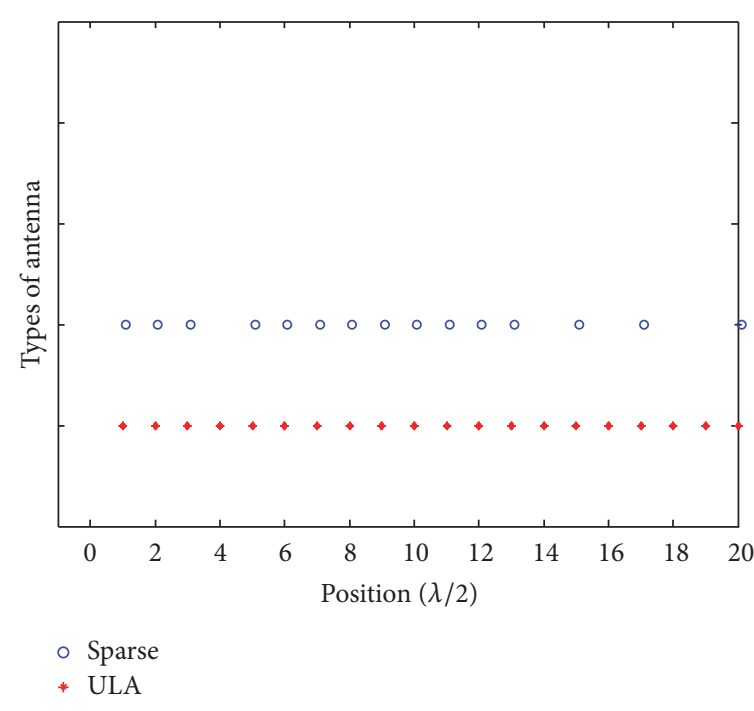

(a) Position of antenna

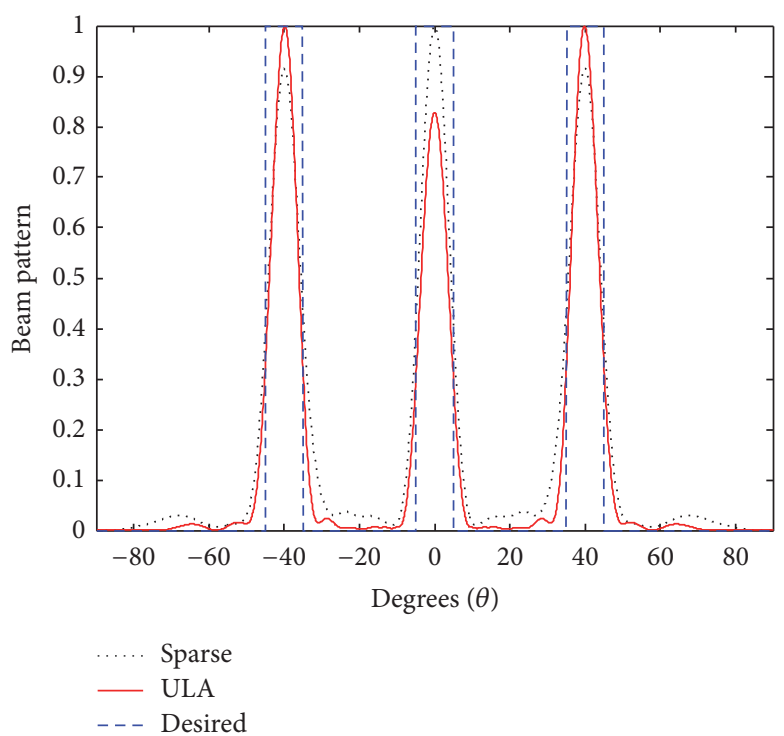

(b) Transmitting beam pattern

FIGURE 5: Results of DE with mutation strategy 2. 


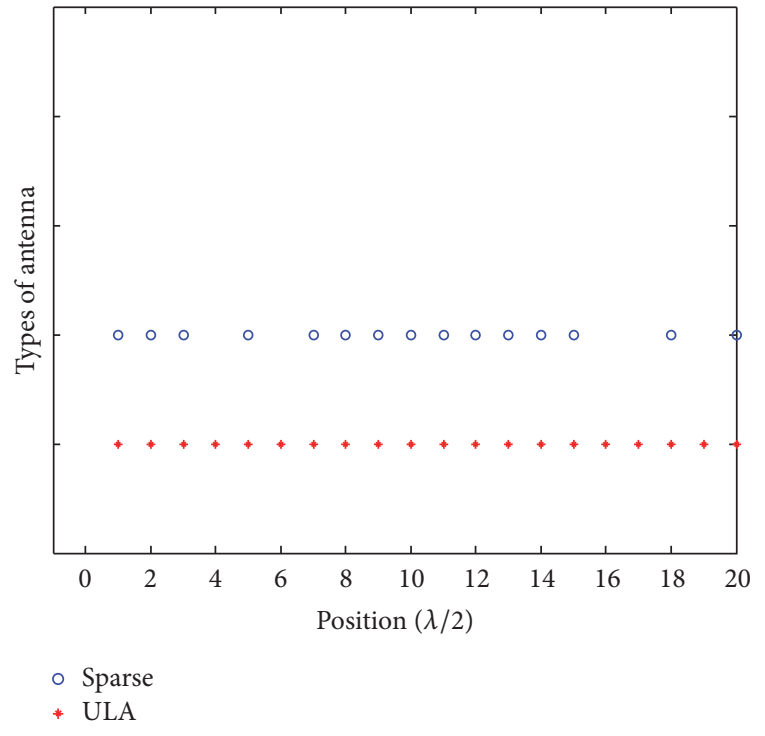

(a) Position of antenna

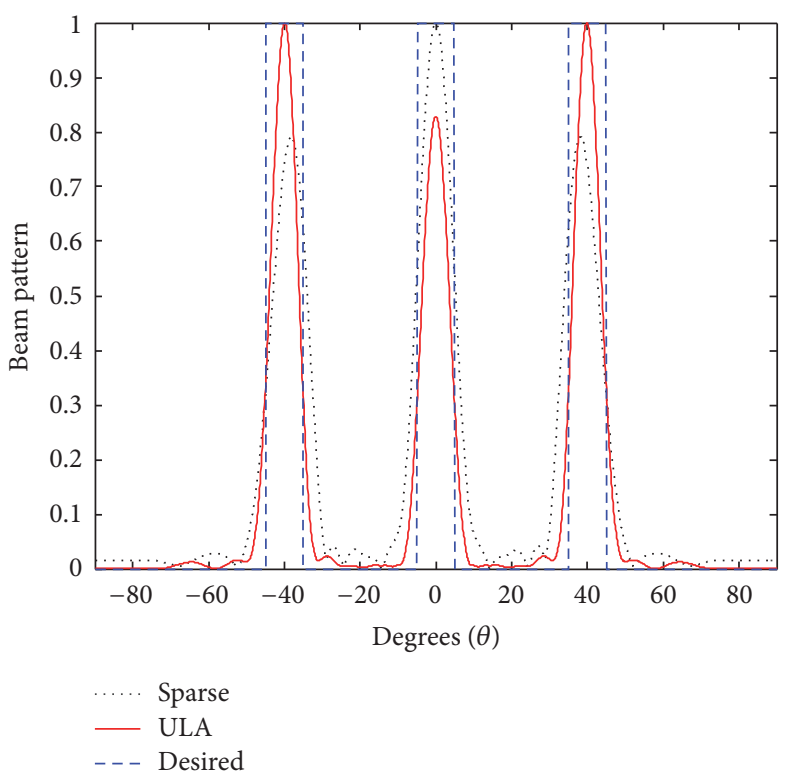

(b) Transmitting beam pattern

Figure 6: Results of DE with mutation strategy 3.

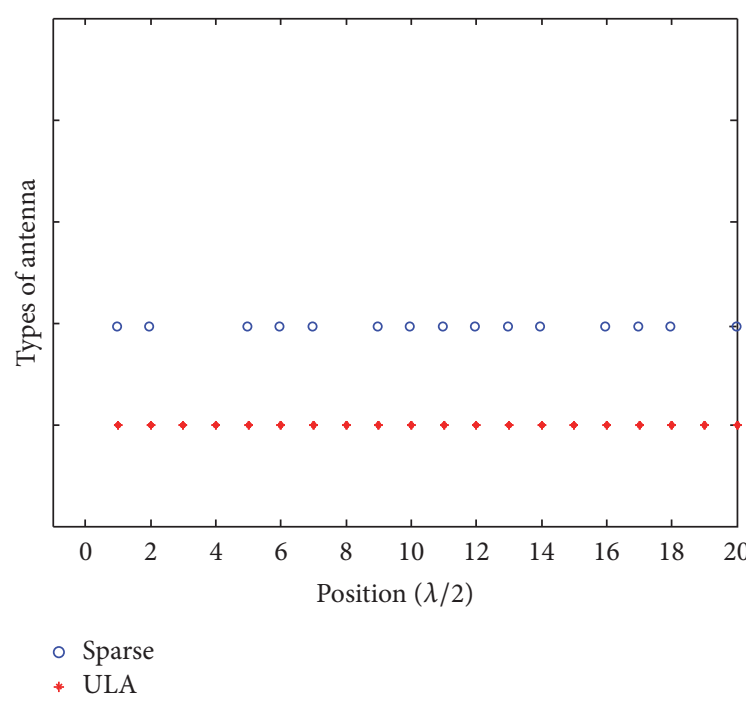

(a) Position of antenna

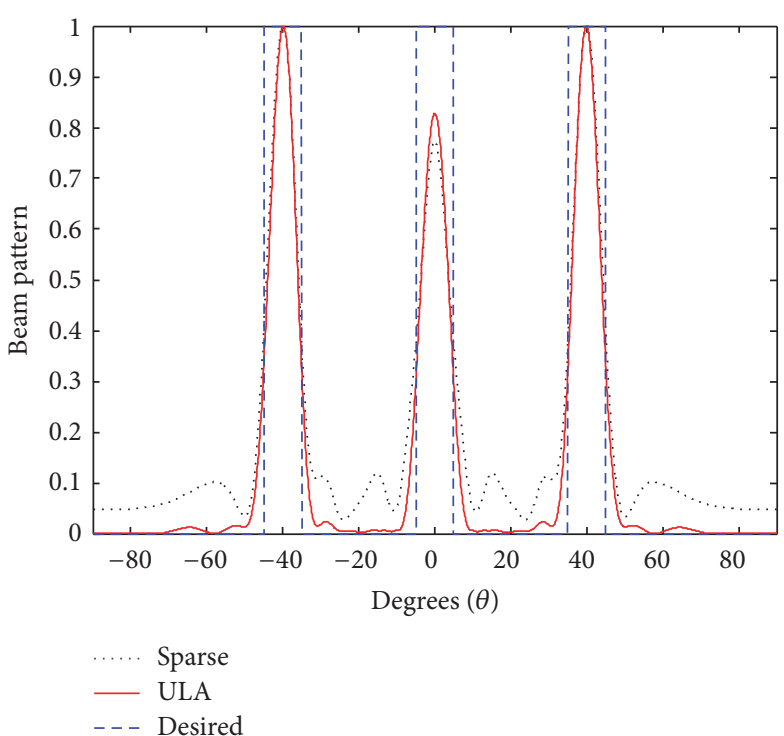

(b) Transmitting beam pattern

FIGURE 7: Results of DE with mutation strategy 4.

between the designed and desired beam patterns in the area of the main lobes reaches perfection. The restriction for the transmitting beam pattern here is to maintain the best approximation in the whole area while keeping the side lobe level as flat as possible as shown in Figures 5, 6, and 8. The violated beam patterns can be found in Figures 4 and 7 .

To investigate the capability and efficiency of $\mathrm{DE}$ algorithms with different mutation strategies for sparse antenna array design, we compare the five strategies in terms of their
MSE, PSLL, iterations needed for convergence, and runtime in Table 2.

Except for the DE with strategy 1, all of the above algorithms can converge to the final result $(\mathrm{MSE}=0.0920)$ after no more than 10 iterations. Although all of these algorithms obtain nearly similar MSE values, their PSLLs greatly differ. To verify their reliability, these algorithms are tested in 10 independent runs. Table 3 presents the PSLLs of each algorithm in 10 runs. 


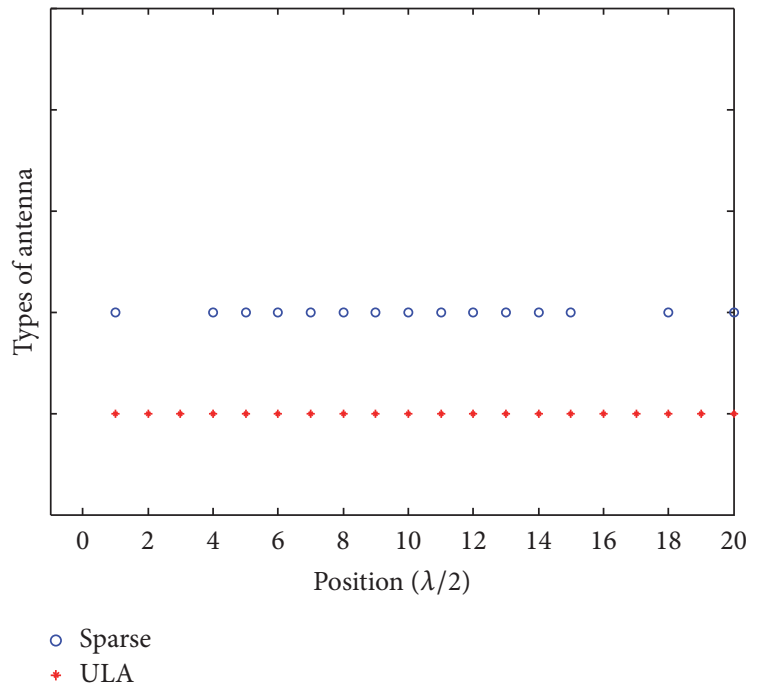

(a) Position of antenna

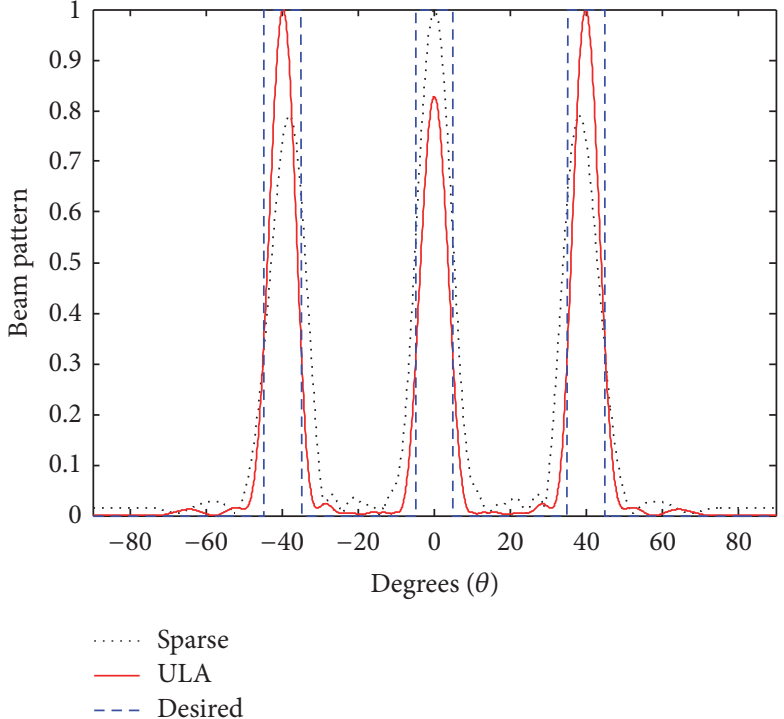

(b) Transmitting beam pattern

Figure 8: Results of DE with mutation strategy 5.

TABLE 2: Comparison of different strategies.

\begin{tabular}{|c|c|c|c|c|}
\hline \multirow{2}{*}{ Algorithm } & \multicolumn{4}{|c|}{ Performance } \\
\hline & MSE & PSLLs & Iterations & Runtime (min/s) \\
\hline DE with strategy 1 & 0.0930 & 0.2291 & 7 & $6 / 59$ \\
\hline DE with strategy 2 & 0.0920 & 0.0296 & 6 & $6 / 50$ \\
\hline DE with strategy 3 & 0.0920 & 0.0719 & 7 & $6 / 52$ \\
\hline DE with strategy 4 & 0.0920 & 0.1196 & 8 & $6 / 54$ \\
\hline DE with strategy 5 & 0.0920 & 0.0417 & 10 & $6 / 51$ \\
\hline
\end{tabular}

TABLE 3: PSLLs in 10 runs.

\begin{tabular}{lcccccccccc}
\hline \multirow{2}{*}{ Algorithm } & \multicolumn{1}{c}{ 1st } & 2nd & 3rd & 4th & 5th & 6th & 7th & 8th & 9th & 10th \\
\hline DE with strategy 1 & 0.228 & 0.063 & 0.165 & 0.095 & 0.048 & 0.105 & 0.024 & 0.323 & 0.166 & 0.089 \\
DE with strategy 2 & 0.021 & 0.112 & 0.133 & 0.051 & 0.014 & 0.078 & 0.051 & 0.058 & 0.206 & 0.118 \\
DE with strategy 3 & 0.092 & 0.053 & 0.027 & 0.065 & 0.037 & 0.058 & 0.088 & 0.029 & 0.0815 & 0.125 \\
DE with strategy 4 & 0.120 & 0.143 & 0.047 & 0.084 & 0.080 & 0.085 & 0.111 & 0.313 & 0.109 & 0.076 \\
DE with strategy 5 & 0.042 & 0.063 & 0.039 & 0.161 & 0.322 & 0.142 & 0.097 & 0.072 & 0.058 & 0.067 \\
\hline
\end{tabular}

The PSLLs are unstable, uncertain, and random in the repeated operation, which suggests that changing mutation strategy cannot keep PSLL as a restriction because the PSLLs of the sparse antenna array design must not be directly constrained. Moreover, the weak constraint of MSE cannot guarantee the suppression of PSLLs at a specific region.

\subsection{Example 3: Sparse Antenna Array Design Using MODE in} the Second Stage. As mentioned in Section 3.2, the MODE algorithm is applied for sparse antenna array design. The results are shown in Figure 9.

The red asterisks in Figure 9 represent the infeasible solution set, while the black diamonds represent the feasible solution set. To achieve the best approximation performance, we choose the minimum value of MSE (MSE $=0.0920)$ in the feasible solution set. The PSLLs obtained using MODE outperform those obtained using DE or GA, while the best matching performance is maintained with respect to the lowest MSE values. Figure 10 shows the corresponding sparse antenna array and beam pattern. Obviously, we can see that this beam pattern is satisfied with our restriction and reflects the performance in Figure 9. Ten independent runs are also conducted in this example.

Table 4 summarizes the results of MODE after 10 runs. The highest PSLL is below 0.02, while all PSLLs slightly fluctuate around 0.0085, which suggests that the PSLLs 
TABle 4: Performance of MODE in 10 runs.

\begin{tabular}{|c|c|c|c|c|c|c|c|c|c|c|}
\hline \multirow{2}{*}{ Algorithm: MODE } & \multicolumn{10}{|c|}{ Index of runs } \\
\hline & 1st & 2nd & 3rd & 4 th & 5 th & 6 th & 7 th & 8th & 9th & 10th \\
\hline MSE & 0.092 & 0.092 & 0.092 & 0.0928 & 0.092 & 0.092 & 0.092 & 0.092 & 0.0920 & 0.0920 \\
\hline PSLLs & 0.0085 & 0.0085 & 0.0108 & 0.0097 & 0.0085 & 0.0085 & 0.0085 & 0.0085 & 0.0085 & 0.0108 \\
\hline Iterations & 27 & 27 & 27 & 27 & 27 & 27 & 28 & 25 & 28 & 26 \\
\hline Runtime & $6 / 00$ & $6 / 05$ & $7 / 57$ & $6 / 20$ & $6 / 25$ & $7 / 23$ & $6 / 12$ & $7 / 00$ & $6 / 37$ & $6 / 28$ \\
\hline
\end{tabular}

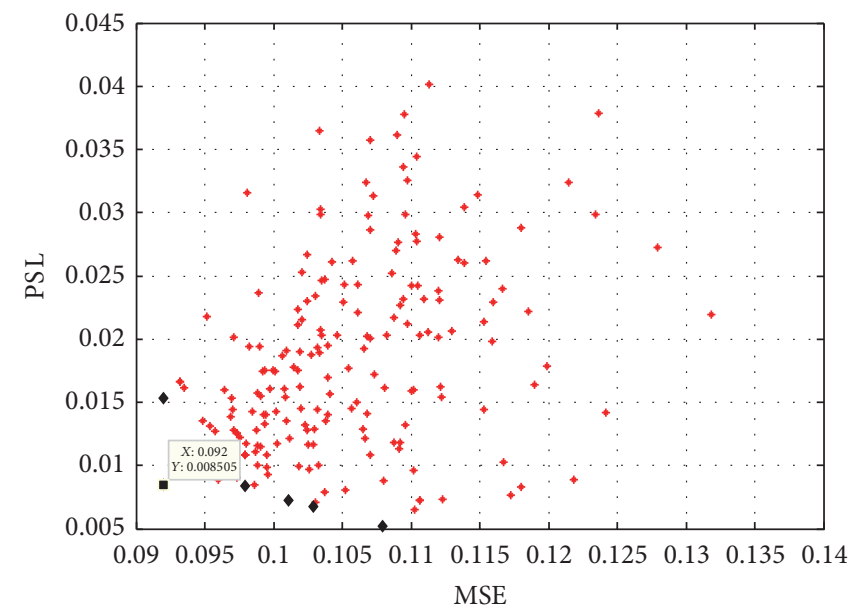

FIgure 9: Pareto fronts produced by MODE.

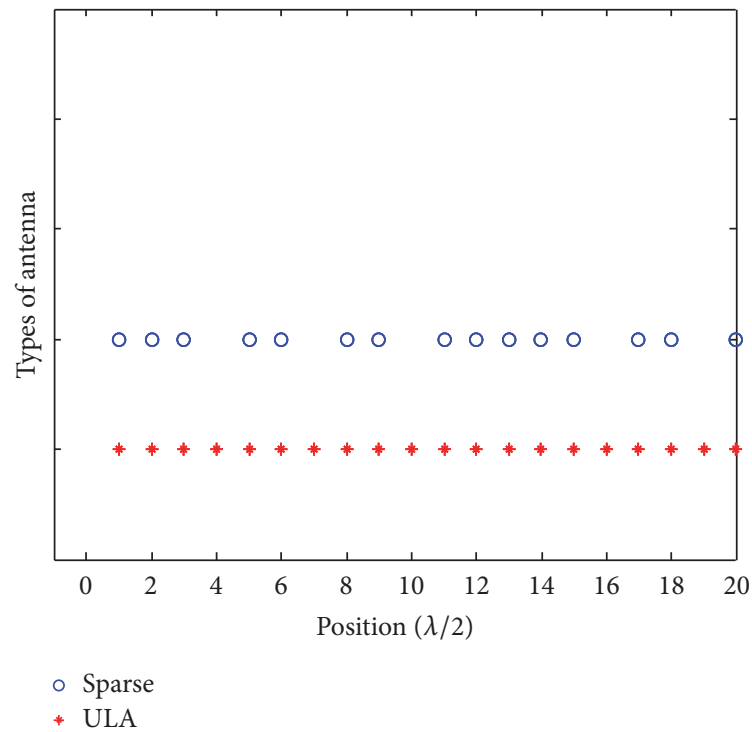

(a) Position of antenna

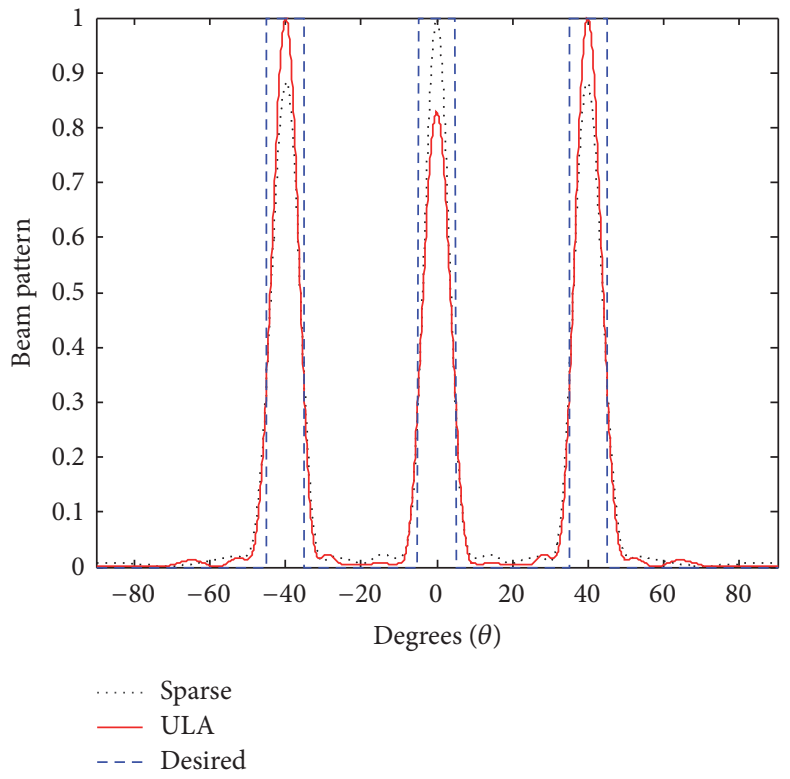

(b) Transmitting beam pattern

Figure 10: Results of MODE.

obtained using MODE are more stable than those obtained using DE. MODE has almost the same running time as DE even though the former adds a constraint to the optimization problem. Moreover, MODE has a simpler algorithm structure than DE. These arguments justify our effort to prevent PSLLs.

\section{Conclusions}

A novel two-stage design approach for the sparse antenna array design of MIMO radar is proposed in this work. CA is applied in the first stage, and two evolution algorithms for 
sparse antenna arrays with different purposes are proposed in the second stage. DE is introduced to design a sparse antenna array aimed at beam pattern synthesis. DE can generate a sparse antenna array for MIMO radar that satisfies beam pattern approximation without producing grating lobes. As a single-objective optimization algorithm, DE faces some challenges in controlling PSLLs. To overcome this drawback, MODE based on Pareto technique is proposed, and PSLL suppression is presented as another objective function to be optimized. The simulation results reveal that MODE outperforms both DE and GA in terms of PSLL while maintaining the same approximation performance and almost the same runtime.

\section{Competing Interests}

The authors declare no conflict of interests regarding the publication of this article.

\section{Acknowledgments}

This work was supported by the National Natural Science Foundation of China under Grants 61371181 and 61501142.

\section{References}

[1] D. R. Fuhrmann and G. S. San Antonio, "Transmit beamforming for MIMO radar systems using signal cross-correlation," IEEE Transactions on Aerospace and Electronic Systems, vol. 44, no. 1, pp. 171-186, 2008.

[2] J. Li and P. Stoica, "MIMO radar with colocated antennas," IEEE Signal Processing Magazine, vol. 24, no. 5, pp. 106-114, 2007.

[3] H. Xu, R. S. Blum, J. Wang, and J. Yuan, "Colocated MIMO radar waveform design for transmit beampattern formation," IEEE Transactions on Aerospace and Electronic Systems, vol. 51, no. 2, pp. 1558-1568, 2015.

[4] W. Roberts, L. Xu, J. Li, and P. Stoica, "Sparse antenna array design for MIMO active sensing applications," IEEE Transactions on Antennas and Propagation, vol. 59, no. 3, pp. 846-858, 2011.

[5] R. Bhattacharya, T. K. Bhattacharyya, and R. Garg, "Position mutated hierarchical particle swarm optimization and its application in synthesis of unequally spaced antenna arrays," IEEE Transactions on Antennas and Propagation, vol. 60, no. 7, pp. 3174-3181, 2012.

[6] K. Chen, X. Yun, Z. He, and C. Han, "Synthesis of sparse planar arrays using modified real genetic algorithm," IEEE Transactions on Antennas and Propagation, vol. 55, no. 4, pp. 1067-1073, 2007.

[7] J. He, D.-Z. Feng, N. H. Younan, and X.-M. Li, “Optimizing thinned antenna array geometry in MIMO radar systems using multiple genetic algorithm," in Proceedings of the IEEE CIE International Conference on Radar (Radar '11), pp. 1178-1187, October 2011.

[8] D. Chen, B. Chen, and S. Zhang, "Multiple-input multipleoutput radar and sparse array synthetic impulse and aperture radar," in Proceedings of the CIE International Conference on Radar (ICR '06), pp. 1-4, Shanghai, China, October 2006.

[9] T. Takayama, M. Sugano, and Y. Tokieda, "Hybrid SIMO and MIMO sparse array radar," in Proceedings of the European Radar Conference (EuRAD '13), pp. 25-28, 2013.
[10] Z.-K. Chen, J. Wang, X.-L. Qiao, and M. Yan, "Waveform design for MIMO radar with sparse antenna array," in Proceedings of the IET International Radar Conference, pp. 1-5, Hangzhou, China, October 2015.

[11] C. Lin, A. Qing, and Q. Feng, "Synthesis of unequally spaced antenna arrays by using differential evolution," IEEE Transactions on Antennas and Propagation, vol. 58, no. 8, pp. 2553-2561, 2010.

[12] D. G. Kurup, M. Himdi, and A. Rydberg, "Synthesis of uniform amplitude unequally spaced antenna arrays using the differential evolution algorithm," IEEE Transactions on Antennas and Propagation, vol. 51, no. 9, pp. 2210-2217, 2003.

[13] S. K. Goudos, K. Siakavara, T. Samaras, E. E. Vafiadis, and J. N. Sahalos, "Sparse linear array synthesis with multiple constraints using differential evolution with strategy adaptation," IEEE Antennas and Wireless Propagation Letters, vol. 10, pp. 670-673, 2011.

[14] R. Farmani and J. A. Wright, "Self-adaptive fitness formulation for constrained optimization," IEEE Transactions on Evolutionary Computation, vol. 7, no. 5, pp. 445-455, 2003.

[15] A. Angantyr, J. Andersson, and J.-O. Aidanpaa, "Constrained optimization based on a multiobjective evolutionary," in Proceedings of the Congress on Evolutionary Computation, vol. 3, pp. 1560-1567, 2003.

[16] P. Stoica, J. Li, and Y. Xie, "On probing signal design for MIMO radar," IEEE Transactions on Signal Processing, vol. 55, no. 8, pp. 4151-4161, 2007.

[17] A. K. Qin, V. L. Huang, and P. N. Suganthan, "Differential evolution algorithm with strategy adaptation for global numerical optimization," IEEE Transactions on Evolutionary Computation, vol. 13, no. 2, pp. 398-417, 2009.

[18] Z. Cai and Y. Wang, "A multiobjective optimization-based evolutionary algorithm for constrained optimization," IEEE Transactions on Evolutionary Computation, vol. 10, no. 6, pp. 658-675, 2006.

[19] S. Li, "Grate lobes/side lobes suppression for sparse array design by using genetic algorithms," in Proceedings of the 2nd International Conference on Innovations in Bio-inspired Computing and Applications (IBICA '11), pp. 371-373, Shenzhen, China, December 2011.

[20] F. Xue, A. C. Sanderson, and R. J. Graves, "Pareto-based multiobjective differential evolution," in Proceedings of the 2003 Congress on Evolutionary Computation (CEC '03), vol. 2, pp. 862-869, Canberra, Australia, December 2003.

[21] M. Ali, P. Siarry, and M. Pant, "An efficient differential evolution based algorithm for solving multi-objective optimization problems," European Journal of Operational Research, vol. 217, no. 2, pp. 404-416, 2012.

[22] S. K. Goudos and J. N. Sahalos, "Pareto optimal microwave filter design using multiobjective differential evolution," IEEE Transactions on Antennas and Propagation, vol. 58, no. 1, pp. 132-144, 2010.

[23] “CVX Research Inc: sedumi_1.3 toolbox,” 2014, http://cvxr.com. 


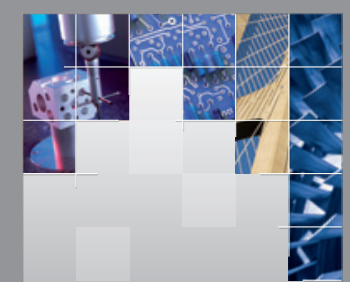

\section{Enfincering}
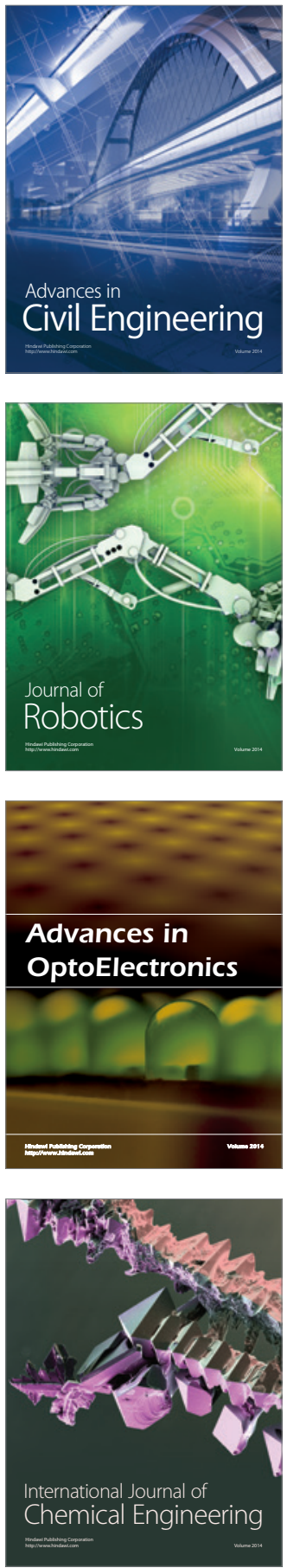

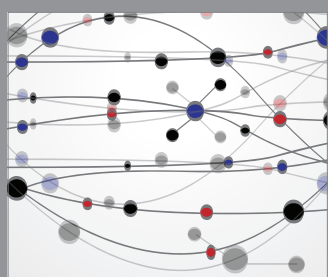

The Scientific World Journal

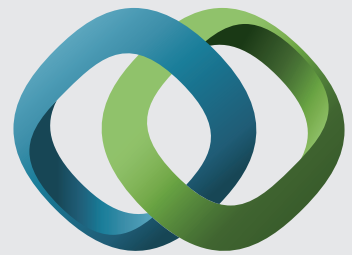

\section{Hindawi}

Submit your manuscripts at

http://www.hindawi.com
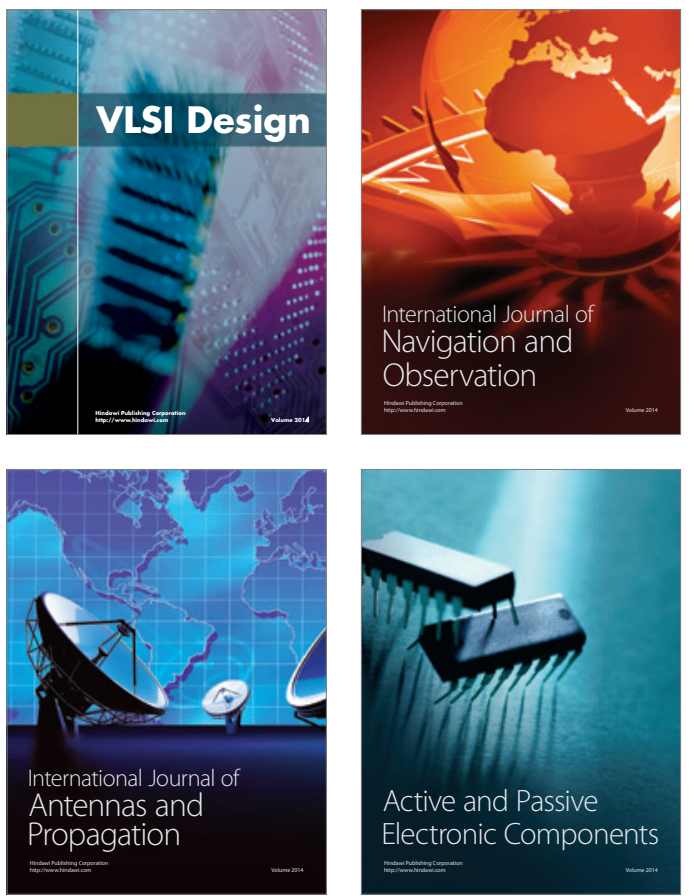
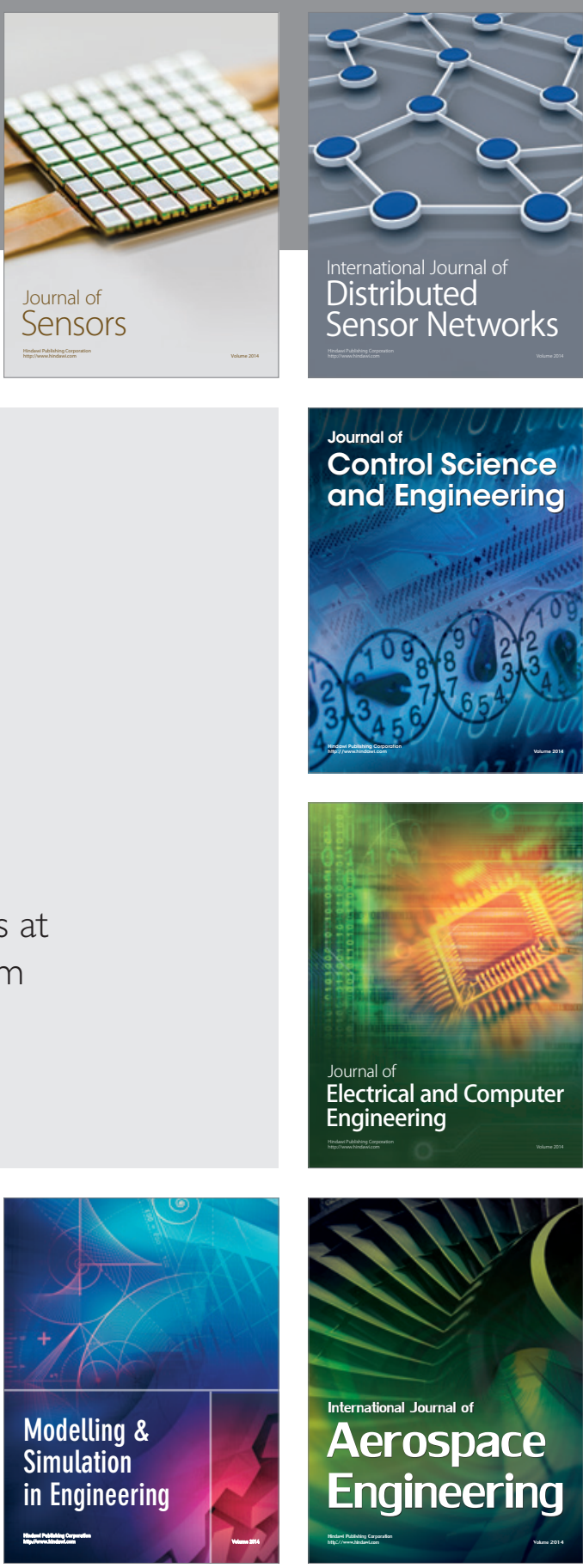

International Journal of

Distributed

Sensor Networks

Journal of

Control Science

and Engineering
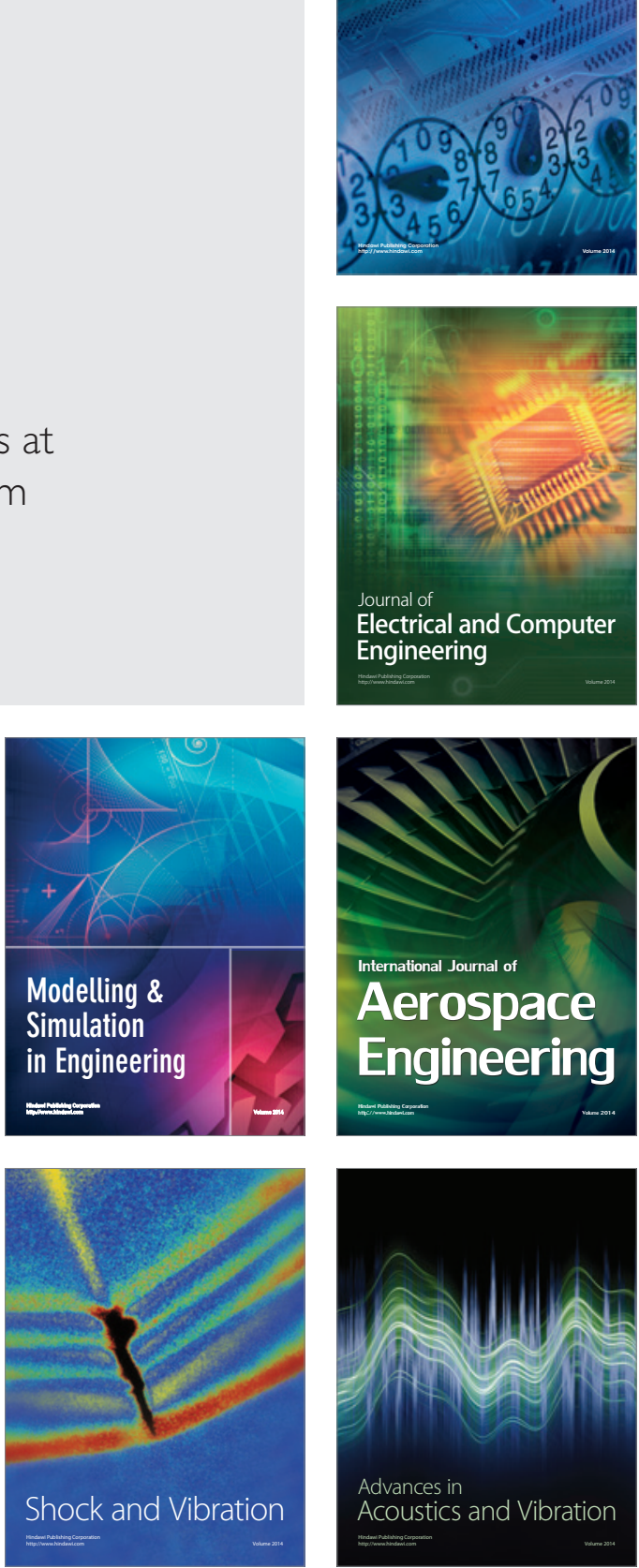